\title{
Projeto "a escola promovendo hábitos alimentares saudáveis": comparação de duas estratégias de educação nutricional no Distrito Federal, Brasil
}

\author{
"Promotion of healthy eating habits by schools" \\ study: comparison of two nutrition education \\ strategies in the Federal District of Brazil
}

Renata Tiene de Carvalho YOKOTA'

Tatiana França de VASCONCELOS ${ }^{1}$

Anelise Rizzolo de Oliveira PINHEIRO2

Bethsáida de Abreu Soares SCHMITZ²

Denise Costa COITINHO 2,3,4

Maria de Lourdes Carlos Ferreirinha RODRIGUES²

RE S U M O

\section{Objetivo}

Analisar e comparar o conhecimento sobre nutrição de professores e alunos do Projeto "a escola promovendo hábitos alimentares saudáveis", submetidos a duas estratégias de educação nutricional em escolas do Distrito Federal.

\section{Métodos}

O Projeto Escola utilizou duas estratégias de intervenção em educação nutricional: intervenção nutricional (A) ações educativas realizadas na comunidade escolar pela equipe; e intervenção nutricional (B) ações educativas desenvolvidas por professores que frequentaram oficinas de capacitação. O conhecimento das crianças foi avaliado por meio de um instrumento sobre pirâmide dos alimentos e um sobre higiene pessoal; sendo aplicado com os professores um questionário do tipo verdadeiro ou falso.

1 Universidade de Brasília, Faculdade de Ciências da Saúde, Departamento de Nutrição, Programa de Pós-Graduação em Nutrição Humana. Campus Universitário Darcy Ribeiro, 70910-900, Brasília, DF, Brasil. Correspondência para/Correspondence to: R.T.C.YOKOTA. E-mail: <renatatiene@yahoo.com.br>.

2 Universidade de Brasília, Observatório de Políticas de Segurança Alimentar e Nutrição. Brasília, DF, Brasil.

3 Programa Mundial de Alimentação. Roma, Itália.

4 Organização Mundial da Saúde. Genebra, Suíça. 
38 | R.T.C. YOKOTA et al.

\section{Resultados}

Foram avaliadas 4 escolas com intervenção A (180 alunos e 19 professores) e 4 com intervenção B (129 alunos e 16 professores). A média de acertos no instrumento de pirâmide dos alimentos foi de 3,2 e 2,9 $(p=0,37)$, respectivamente pelas crianças que receberam as intervenções nutricionais $A$ e $B$. Na atividade sobre higiene pessoal, para estes mesmos alunos, observaram-se percentuais de acerto de $61,1 \%$ e $73,6 \%(p=0,10)$. Os professores que receberam as intervenções nutricionais $A$ e $B$ obtiveram percentuais de acertos semelhantes nos questionários, em torno de 90,0\% $(p=0,06)$.

\section{Conclusão}

Observou-se que os dois tipos de intervenção de educação nutricional foram igualmente úteis para promover o conhecimento de alunos e professores. Portanto, faz-se necessário incentivar a realização de processos de educação nutricional no ambiente escolar, de acordo com a diretriz da Política Nacional de Alimentação e Nutrição que diz respeito à promoção da saúde.

Termos de indexação: Avaliação de processo educativo. Educação alimentar e nutricional. Escola. Nutrição. Promoção da alimentação saudável.

\section{A B S T R A C T}

\section{Objective}

The objective of this study was to analyze and compare the nutrition knowledge of teachers and students who participated in the study "Promotion of healthy eating habits by schools" and were submitted to two different strategies of nutrition education in schools of the Federal District, Brazil.

\section{Methods}

The study administered two strategies of nutrition education intervention: nutrition intervention (A) administration of educational activities in the school community by the research team; and nutrition intervention (B) educational activities were administered by teachers who participated in the training workshops. The knowledge of the students was assessed by an instrument based on the food pyramid and another one based on personal hygiene. The teachers were assessed by means of a true-or-false questionnaire.

\section{Results}

Four schools submitted to nutrition intervention A (180 students and 19 teachers) and four schools submitted to nutrition intervention B (129 students and 16 teachers) were assessed. The average number of correct answers given by the students on the instrument based on the food pyramid was 3.2 for students in intervention $A$ and 2.9 for students in intervention $B(p=0.37)$. On the instrument about personal hygiene for the same students, $61.1 \%$ and $73.6 \%$, respectively, answered it correctly $(p=0.10)$. The teachers submitted to interventions $A$ and $B$ answered a similar number of questions correctly, around $90.0 \%(p=0.06)$.

\section{Conclusion}

This study showed that two different types of nutrition education intervention were equally useful to promote nutrition knowledge among teachers and students. Therefore, it is necessary to promote nutrition education programs in schools, in accordance with the National Food and Nutrition Policies, which regard health promotion strategies.

Indexing terms: Evaluation of educative process. Food and nutrition education. School. Nutrition. Promotion of health eating habits.

\section{N T R O D U ÇÃ O}

A promoção da saúde é considerada uma estratégia importante no processo saúde-doença-cuidado, sendo direcionada para o fortalecimento do caráter promocional e preventivo ${ }^{1}$. Uma das estratégias mais efetivas que contribuem para a promo- ção da saúde é a combinação de apoio educacional e ambiental, envolvendo dimensões não só individuais, mas também organizacionais e coletivas, visando, dessa forma, atingir ações e condições de vida conducentes à saúde 2 . Nesse sentido, a educação nutricional pode ser considerada um componente decisivo na promoção de saúde ${ }^{3,4}$. 
Nessa perspectiva, a escola é um espaço privilegiado para a construção e a consolidação de práticas alimentares saudáveis em crianças, pois é um ambiente no qual atividades voltadas à educação em saúde podem apresentar grande repercussão $0^{5-8}$. Nesse contexto estão inseridas todas as dimensões do aprendizado: ensino, relações lar-escola-comunidade e ambientes físico e emocional, podendo assim beneficiar os estudantes em fases fundamentais de suas vidas: infância e adolescência9-11.

A promoção da alimentação saudável no ambiente escolar parte de uma visão integral e multidisciplinar do ser humano, que considera as pessoas, principalmente o escolar, em seu contexto familiar, comunitário e socia|8,12. Procura também, desenvolver conhecimentos, habilidades e destrezas para o auto-cuidado da saúde e a prevenção das condutas de risco em todas as oportunidades educativas. Além disso, fomenta uma análise crítica e reflexiva sobre os valores, as condutas, as condições sociais e os estilos de vida, buscando fortalecer tudo aquilo que contribui para melhoria da saúde e do desenvolvimento humano $^{13}$. Adicionalmente, órgãos internacionais vêm recomendando fortemente práticas de promoção de saúde no ambiente escolar. Isto tem sido feito principalmente para crianças maiores de cinco anos que, apesar da suscetibilidade biológica, nutricional e social, em geral, se acham excluídas das prioridades estratégicas das políticas de saúde ${ }^{3}$.

A fase da infância apresenta importantes aspectos para a formação de hábitos e práticas comportamentais em geral, e especificamente alimentares ${ }^{8,12}$. Inserida no contexto familiar, a criança começa a formar e internalizar os padrões de comportamento alimentar, em termos de escolha e quantidade de alimentos, horário e ambiente das refeições ${ }^{6}$. Trata-se de um processo que se inicia nesta fase e se estende por todas as demais fases do ciclo de vida ${ }^{5,14}$.

Este momento é importante para o desenvolvimento da consciência crítica, de forma a propiciar a aplicação de práticas para promoção, manutenção e até recuperação da própria saúde e da comunidade da qual a criança faz parte, bem como capacitá-la a reivindicar meios para sua melhoria3,7,13,15. Nesse sentido, observa-se que a prevalência de obesidade tem aumentado em todos os segmentos sociais e nas diversas faixas etárias, como resultado do elevado consumo de alimentos de alta densidade energética e da redução da prática de atividade física. O fenômeno da transição nutricional atinge também a população infantil, sendo caracterizado, atualmente, pelo aumento do consumo de alimentos ricos em gordura, sal e açúcares refinados e pobres em micronutrientes e fibras, como os refrigerantes, lanches tipo fast food, balas, chocolates, frituras, sorvetes, entre outros ${ }^{16-18}$.

A estratégia de promoção da saúde na escola envolve vários atores sociais, como alunos, professores, coordenadores, donos de cantinas e pais ou responsáveis, permitindo uma abordagem dialógica da produção do conhecimento no ambiente escolar ${ }^{3,12}$. A capacitação de professores e de todos os outros atores sociais que compõem a comunidade escolar potencializa mudanças no ambiente escolar, as quais dão suporte para adoção de hábitos alimentares saudáveis ao longo da vida9,19-21.

Para que as atividades de educação em saúde sejam bem desenvolvidas na escola, os professores, atores chaves desse processo, que atuam como facilitadores da articulação entre teoria e situação prática, precisam estar bem informados e orientados sobre o tema, para reconhecer a importância de sua atuação na área de saúde ${ }^{22-24}$. O treinamento, a sensibilização e a motivação dos professores nestas atividades podem ser mediados por profissional da área da saúde ${ }^{3}$.

O Projeto "A escola promovendo hábitos alimentares saudáveis" existe no Distrito Federal desde 2001, com o objetivo de promover a alimentação saudável no ambiente escolar, testando metodologias e estratégias que possibilitem sua 
reprodutibilidade em outros locais e contextos, envolvendo escolas públicas e privadas de Ensino Infantil e Fundamental. O Projeto é vinculado ao Observatório de Segurança Alimentar e Nutrição da Universidade de Brasília, o qual tem a promoção da alimentação saudável como uma de suas linhas de investigação.

Assim, este estudo teve como objetivo analisar e comparar o conhecimento sobre nutrição de professores e alunos participantes do Projeto "A escola promovendo hábitos alimentares saudáveis", submetidos a duas estratégias de educação nutricional.

\section{M É T O D O S}

Este Projeto foi idealizado e iniciado no ano de 2001, sendo que a partir de 2003 foi reestruturado em suas estratégias de ação, visando potencializar a promoção de práticas alimentares saudáveis em escolas de Ensino Infantil e Fundamental, público e privado do Distrito Federal, buscando proporcionar uma vida mais saudável à comunidade escolar, incluindo o corpo são, o modo de vida de cada um, a cultura e os valores e crenças $^{11}$.

O Projeto Escola utilizou duas estratégias de intervenção em educação nutricional nas escolas participantes. A categorização dessas intervenções foi feita com base em diferentes tipos de atividades realizadas:

- Intervenção nutricional A: as ações educativas com a comunidade escolar foram desenvolvidas pela equipe do projeto, por meio de palestras educativas realizadas nas escolas. Assim, todas as turmas de Ensino Infantil e Fundamental das escolas participantes foram mobilizadas pelas atividades de educação nutricional.

- Intervenção nutricional $B$ : as ações educativas com a comunidade escolar foram desenvolvidas por professores que frequentaram oficinas temáticas de capacitação ${ }^{11}$. Uma vez que apenas os professores que se interessaram em se inscrever nas oficinas foram capacitados, o processo de educação nutricional tendeu a se concentrar apenas nas turmas dos professores participantes.

Neste estudo, foram avaliadas quatro escolas que receberam a intervenção nutricional $A$, entre as sete participantes. Destas escolas, uma era particular e três eram públicas, sendo uma destas rural. $\mathrm{Na}$ intervenção nutricional $\mathrm{B}$, cinco escolas participariam, entretanto, uma foi excluída da avaliação devido à sua metodologia de ensino, que não aceitou a utilização de instrumentos pré-elaborados com seus alunos. Foram avaliados os alunos e professores de Ensino Infantil e Fundamental nos dois tipos de intervenção.

A fim de obter a autorização para participação no estudo, os diretores e os pais dos alunos das escolas participantes assinaram um Termo de Consentimento Livre e Esclarecido. Este estudo foi aprovado pelo Comitê de Ética e Pesquisa da Faculdade de Ciências da Saúde da Universidade de Brasília (protocolo 006/2005).

Foram elaborados dois instrumentos quantitativos para avaliação do conhecimento sobre nutrição das crianças, tendo sido os mesmo construídos em um formato preferencial de figuras e jogos, mais atrativo visualmente e de maior facilidade de compreensão para esta faixa etária ${ }^{4-6}$.

- Instrumento sobre a pirâmide dos alimentos: os alunos deveriam relacionar duas colunas de alimentos de acordo com o grupo alimentar correspondente da pirâmide dos alimentos (Anexo 1). Foram avaliados os grupos das frutas, o grupo das carnes e ovos, o grupo das hortaliças e o grupo dos cereais, pães e massas.

- Instrumento sobre a higiene das mãos antes da realização de refeições: foi composto por uma história em quadrinhos na qual as crianças deveriam ordenar corretamente os eventos, enumerando-os, a saber: brincar, chegar da escola, lavar as mãos e realizar a refeição (Anexo 2). Foi considerada correta qualquer sequência que enumerasse a lavagem das mãos antes da realização da refeição. 
A equipe de avaliação do Projeto Escola foi responsável pela aplicação dos instrumentos de avaliação das crianças em cada uma das escolas participantes. Os dois instrumentos foram aplicados no mesmo dia. A fim de garantir o entendimento das crianças em relação aos instrumentos, um integrante do projeto leu as instruções e verificou-se se os alunos compreendiam o significado dos desenhos dos instrumentos em cada uma das classes participantes. Com esta conduta, foi possível evitar que os professores auxiliassem os seus alunos a responder os instrumentos.

Para avaliação dos conhecimentos dos professores foi elaborado um questionário do tipo verdadeiro ou falso sobre assuntos abordados nas palestras educativas realizadas pela equipe do projeto nas escolas (intervenção nutricional A) e nas oficinas de capacitação (intervenção nutricional B). O questionário continha nove itens: cinco sobre alimentação saudável, três sobre pirâmide dos alimentos e um sobre higiene alimentar (Anexo 3). Os professores responderam ao questionário no último dia de participação das oficinas de capacitação ou palestras realizadas na própria escola.

A atividade de avaliação descrita neste estudo foi realizada no período de janeiro de 2004 a dezembro de 2005, por quatro nutricionistas contratadas para tal, que não faziam parte da equipe fixa do Projeto. Portanto, havia uma distinção entre a equipe que executou as ações educativas e a equipe que avaliou estas mesmas ações.

Foi realizado teste $t$ de Student para comparação da média do número de acertos nos instrumentos dos professores e das crianças das escolas com intervenção nutricional A e B. Além deste teste, foram feitos os testes Qui-quadrado e o exato de Fisher. O resultado foi considerado significante quando $p<0,05$.

\section{RES U L T A D O S}

Foram avaliados 180 alunos das escolas submetidas à intervenção nutricional A, sendo
$88 \%$ do ensino fundamental, e 129 alunos das escolas submetidas à intervenção nutricional $B$, sendo $74 \%$ do ensino fundamental. A faixa etária das crianças avaliadas foi de 5 a 10 anos, sendo a média de idade do Ensino Infantil de 6,3 (Desvio-Padrão - DP=0,9) anos e do Ensino Fundamental (1 ${ }^{\text {a }}$ a $4^{\text {a }}$ série), de 8,9 $(\mathrm{DP}=2,1)$ anos.

No instrumento sobre a pirâmide alimentar, foi avaliado o número de acertos para cada grupo de alimentos nos dois tipos de intervenção (Figura 1). Foi observada maior diferença nos percentuais de acertos relativos ao grupo de pães, cereais e massas (diferença de 18,6\%) e ao de carnes e ovos (diferença de 11,7\%). Entretanto, a média final de acertos foi similar nos dois tipos de intervenção $(p=0,37)$.

$\mathrm{Na}$ atividade da história em quadrinhos, que tratava da higienização das mãos antes da realização das refeições, também não foi verificada diferença estatisticamente significante entre os dois tipos de intervenção $(p=0,10)$.

O questionário para avaliação dos professores foi aplicado em 19 professores que receberam a intervenção nutricional $A$ e em 16 que receberam a intervenção $B$ (Tabela 1). Verificou-se que dos 9 itens avaliados, apenas dois apresentaram diferença significante entre os percentuais de acertos dos professores nos dois tipos de intervenção: no item relativo à classificação do milho no grupo alimentar da pirâmide dos alimentos, e no item referente a inclusão da higiene dos alimentos no conceito de alimentação saudável (Figura 2). No cômputo final, as médias de acerto foram similares $(p=0,06)$.

\section{I S C U S S Ã O}

O processo de avaliação permite analisar os resultados das ações, quantificar a magnitude de seus impactos e elaborar propostas para reorientação das mesmas e aperfeiçoamento da funcionalidade de seus processos ${ }^{8,25}$. Nesse sentido, a avaliação deve estar integrada a todo 
Tabela 1. Percentuais de acertos do questionário de avaliação dos professores, segundo tipos de intervenção nutricional. Distrito Federal, Brasil, 2005.

\begin{tabular}{lcc}
\hline Itens avaliados & Intervenção nutricional A & Intervenção nutricional B \\
\hline Fracionamento das refeições & 100,0 & 93,8 \\
Inclusão de prazer e satisfação no conceito de alimentação saudável & 100,0 & 100,0 \\
Existência de um padrão único de alimentação saudável ideal para todos & 100,0 & 100,0 \\
Classificação do milho dentro dos grupos alimentares da pirâmide dos alimentos & 100,0 & $68,8^{\mathbf{a}}$ \\
Base da alimentação de acordo com a pirâmide de alimentos & 89,5 & 87,5 \\
Frequência de consumo de doces e biscoitos por crianças & 94,7 & 100,0 \\
Higiene de alimentos dentro do conceito de alimentação saudável & 68,4 & $100,0^{\mathbf{a}}$ \\
Relação de alimentação saudável e magreza & 100,0 & 100,0 \\
Relação de exercício físico em excesso e saúde & 84,2 & 93,8 \\
\hline Média de acertos & 93,0 & $93,8^{\mathbf{b}}$ \\
\hline
\end{tabular}

a $p<0,05 ; \quad$ b $p=0,06$.

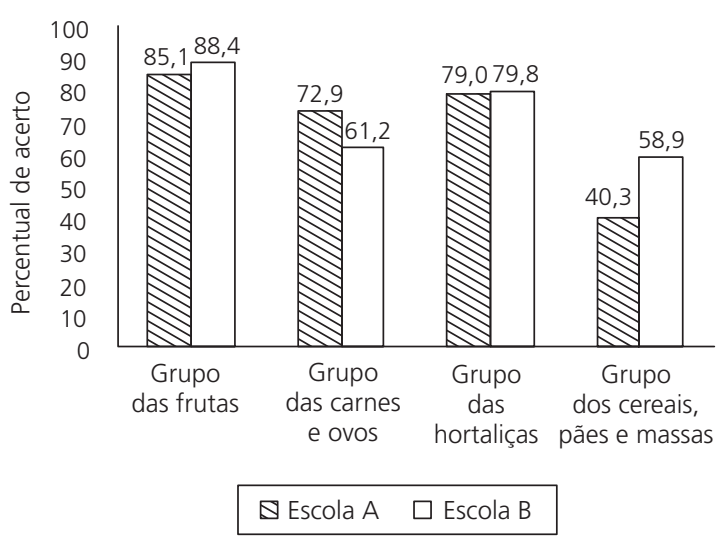

Figura 1. Percentuais de acertos das crianças participantes das intervenções nutricionais $A$ e $B$, quanto ao conhecimento sobre a pirâmide alimentar. Distrito Federal, Brasil, 2005.

Nota: $p=0,37$.

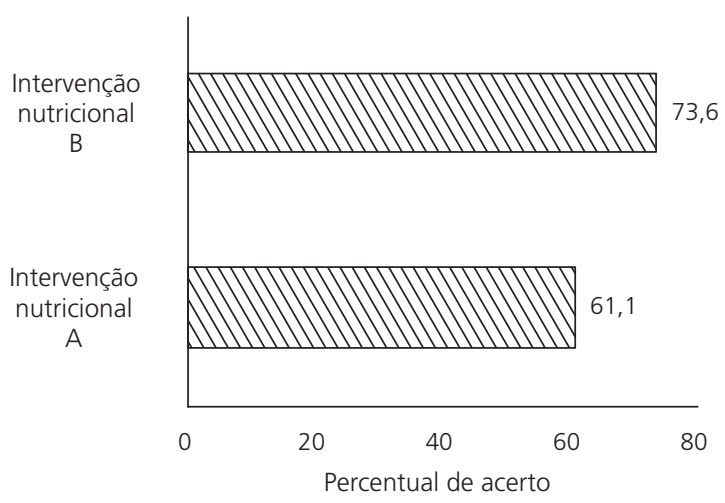

Figura 2. Percentuais de acertos das crianças participantes das intervenções nutricionais A e B na história em quadrinhos (higiene pessoal). Distrito Federal, Brasil, 2005.

Nota: $p=0,10$. o processo, devendo aplicar-se a todos os tipos de intervenção em educação nutricional5,26,27.

Este estudo, procurando avaliar o resultado das distintas estratégias educativas desenvolvidas com alunos e professores pelo Projeto "A Escola Promovendo Hábitos Alimentares Saudáveis", se enquadra neste pressuposto da necessidade de execução de processos avaliativos que propiciem uma reorientação das ações programadas.

Como demonstrado nos resultados, em relação aos alunos submetidos às intervenções nutricionais $A$ e $B$, não foi observada diferença estatisticamente significante entre as médias finais de acerto no instrumento da pirâmide dos alimentos, assim como em cada item do instrumento que correspondeu a um grupo de alimento da pirâmide alimentar. Entretanto, verificou-se menor percentual de acertos para os dois tipos de intervenção na ligação correspondente ao grupo de cereais, pães e massas e ao grupo de carnes e ovos, uma vez que grande parte das crianças associou o pão ao ovo e o macarrão ao frango.

Uma possível explicação para este resultado é o fato de que as crianças, ao relacionarem os alimentos, os associam aos seus próprios hábitos, ou seja, ao que estão acostumadas a comer e não aos nutrientes presentes nestes alimentos, e que determinam a separação dos grupos da pirâmide alimentar ${ }^{8}$. Sabe-se que a alimentação é a primeira aprendizagem social da criança e a imer- 
são da criança em um contexto alimentar, inerente a cada cultura, irá influenciar nas escolhas alimentares $^{7,28}$. Adicionalmente, os alimentos não são vistos apenas como fontes de nutrientes, mas também como símbolos formados por múltiplas experiências, que geram categorias alimentares cotidianamente utilizadas pelos membros de uma sociedade, sem que estes tenham verdadeira consciência disto ${ }^{28}$.

No instrumento da história em quadrinhos não houve diferença estatisticamente significante do número de acertos entre os alunos que receberam as intervenções nutricionais A e B. O elevado percentual de acertos encontrado pode ser devido ao modelo de higiene que a escola exerce para as crianças, incluindo o conceito e o aprendizado sobre higiene pessoal e como lavar as mãos antes das refeições ${ }^{29}$. Destaca-se ainda que o Ministério da Educação sugere que este tema seja incluído nos Parâmetros Curriculares Nacionais, pois pode auxiliar a escola a cumprir o papel de formar alunos cidadãos ${ }^{3}$.

A análise dos resultados obtidos com os alunos indicou que tanto a presença da equipe de nutricionistas na escola, quanto a capacitação de professores realizada nas oficinas desenvolvidas na Universidade de Brasília, parecem ter contribuído efetivamente para a transmissão de conhecimentos sobre nutrição. Verifica-se, portanto, a importância de utilizar processos de educação nutricional para promover a formação de hábitos alimentares saudáveis desde a infância, uma vez que estes são formados nesta fase da vida6,9,19.

Em estudo realizado por Triches \& Giugliani ${ }^{30}$, com crianças das escolas municipais do Rio Grande Sul, foi observado que os escolares com maior índice de massa corporal apresentavam menor conhecimento de nutrição e possuíam práticas alimentares menos saudáveis ${ }^{30}$. Estes resultados sugerem que a realização de intervenções nutricionais no ambiente escolar pode ter papel positivo na prevenção do desenvolvimento de doenças crônicas na vida adulta.
Ao analisar a média de acertos dos professores no questionário aplicado, em geral não foi encontrada diferença estatisticamente significante entre os dois tipos de intervenção nutricional. Neste caso, tanto a realização de atividades de educação nutricional diretamente no ambiente escolar por nutricionistas, quanto a capacitação de professores para que estes se tornassem multiplicadores do conhecimento nutricional aos seus alunos, são capazes de transmitir conhecimentos sobre nutrição de forma semelhante.

O elevado percentual de acertos observado no instrumento de avaliação dos professores reforça a importância da transmissão de conhecimentos sobre nutrição para os mesmos. Entende-se que o professor é considerado o elemento principal no processo de educação nutricional dos alunos, por se encontrar em posição estratégica e em contato diário com os mesmos 8,23,24,29.

Dos nove itens avaliados, em apenas dois foram verificadas diferenças significantes de conhecimento entre os professores submetidos às duas estratégias de intervenção nutricional. Os professores submetidos à intervenção nutricional $B$ apresentaram $69 \%$ de acertos no item relacionado à classificação do milho nos grupos de alimentos da pirâmide alimentar, comparados a 100\% de acertos dos professores que participaram da intervenção nutricional $A(p<0,05)$. Esperava-se que o número de acertos entre os grupos fosse semelhante, uma vez que o item sobre pirâmide dos alimentos foi bastante explorado nas duas modalidades de intervenção nutricional.

Por outro lado, os professores que receberam a intervenção nutricional A tiveram menor percentual de acerto do item 7 do questionário, quando comparados aos professores submetidos à intervenção nutricional $B(p<0,05)$. Este item estava relacionado à inclusão da higiene dos alimentos no conceito de alimentação saudável. Destaca-se que este item é abordado habitualmente pelos professores dentro das disciplinas escolares. Ressalta-se ainda a importância da es- 
cola como colaboradora para a difusão de meios de prevenção e preservação da saúde, que incluem os conceitos de higiene dos alimentos ${ }^{29}$.

\section{O N CLUS Ã O}

Observou-se que tanto a realização de oficinas de capacitação sobre educação nutricional para professores, como a realização direta de palestras e outras atividades pedagógicas para a comunidade escolar pelo nutricionista, são processos igualmente úteis de intervenção nutricional no âmbito escolar.

Dessa forma, ressalta-se a necessidade de incentivar a realização de programas de educação nutricional nas escolas, um vez que foram identificados neste trabalho dois processos de intervenção nutricional que produziram resultados semelhantes. A promoção de práticas alimentares saudáveis está inserida no contexto da adoção de estilos de vida saudáveis, componente importante da promoção da saúde, uma das diretrizes da atual Política Nacional de Alimentação e Nutrição.

Outra análise que pode ser feita em relação a este resultado é a possibilidade de uma definição mais segura da escolha da estratégia de promoção da educação nutricional, de acordo com as condições existentes em cada contexto. Dessa maneira, é possível uma maior potencialização do uso de recursos materiais e humanos sem que isto acarrete prejuízo na aprendizagem. Portanto, as oficinas de capacitação podem ser priorizadas, por oferecerem maior praticidade de ação e menor custo operacional.

Sugere-se que o nutricionista seja o responsável pela intermediação entre os saberes, na medida em que assume a condição de multiplicador de conteúdos e temas em alimentação e nutrição e, por isso, possui um papel determinante no processo de implantação de hábitos alimentares saudáveis na escola.

Entende-se também que o setor público precisa assumir a responsabilidade de fomentar mudanças organizacionais, em termos de políticas públicas coletivas, de forma a favorecer escolhas saudáveis no campo individual. A construção de políticas públicas resultantes de diálogos equânimes entre o Estado, a sociedade, e o mercado parece ser o caminho para a construção de um modo de viver que permita melhores condições de saúde para a população. Assim, são pressupostos da promoção da alimentação saudável, a ampliação e o fomento de estratégias educativas capazes de permitir estas escolhas, como as apresentadas neste estudo.

\section{A GRADECIMENTOS}

À Ana Floresta, Mariana Côrtes e Carolina Seidler, pelo auxílio na coleta de dados, a Janine Coutinho pelo auxílio na coordenação, a Eduardo Silva, pelo auxílio nas análises estatísticas e ao Ministério da Saúde e ao Conselho Nacional de Desenvolvimento Científico e Tecnológico, pelo financiamento.

\section{OLABORADORES}

R.T.C. YOKOTA participou da coleta dos dados, da análise e da interpretação dos resultados e da elaboração do artigo. T.F. VASCONCELOS participou da coleta dos dados, da análise e da interpretação dos resultados. A.R.O. PINHEIRO participou da análise e da interpretação dos resultados e da elaboração do artigo. B.A.S. SCHMITZ e D.C. COITINHO participaram do planejamento da pesquisa e das correções finais do manuscrito. M.L.C.F. RODRIGUES participou da concepção e do desenho da pesquisa e da elaboração do artigo.

REFER Ê N CIAS

1. Santos LAS. Educação alimentar e nutricional no contexto da promoção de práticas alimentares saudáveis. Rev Nutr. 2005; 18(5):681-92. doi: 10.1590/S1415-S2732005000500011.

2. Candeias NMF. Conceitos de educação e de promoção em saúde: mudanças individuais e mudanças organizacionais. Rev Saúde Pública. 1997; 31(2):209-13. doi: 10.1590/S0034-89101 997000200016. 
3. Bizzo MLG, Leder L. Educação Nutricional nos parâmetros curriculares nacionais para o ensino fundamental. Rev Nutr. 2005; 18(5):661-7. doi: 10.1590/S1415-S2732005000500009.

4. Gabriel CG, Santos MV, Vasconcelos FAG. Avaliação de um programa de hábitos alimentares saudáveis em escolares de Florianópolis, Santa Catarina, Brasil. Rev Bras Saúde Mater Infant. 2008; 8(3): 299-308. doi: 10.1590/S1519-382920080003 00009.

5. Pérez-Rodrigo C, Aranceta J. School-based nutrition education: lessons learned and new perspectives. Public Health Nutr. 2001; 4(1A):131-9. doi: 10.1079/PHN2000108.

6. Anderson AS, Porteous LEG, Foster E, Higgins C, Stead $M$, Hetherington $M$, et al. The impact of a school-based nutrition education intervention on dietary intake and cognitive and attitudinal variables relation to fruits and vegetables. Public Health Nutr. 2004; 8(6):650-6. doi: 10.1079/ PHN2004721.

7. Mahr J, Wuestefeld M, Haaf JT, Krawinkel MB. Nutrition education for illiterate children in Southern Madagascar: addressig their needs, perceptions and capabilities. Public Health Nutr. 2004; 8(4):366-72. doi: 10.1079/PHN2004683.

8. Gaglianone CP, Taddei JAAC, Colugnati FAB, Magalhâes CG, Davanço GM, Macedo L, et al. Educação nutricional no ensino público fundamental em São Paulo, Brasil. projeto reeducação aos riscos de adoecer e morrer na maturidade. Rev Nutr. 2006; 19(3):309-20. doi: 10.1590/S1415-S2 732006000300002.

9. Burström B, Haglund BJA, Tillgren P, Berg L, Wallin $E$, Ullén $H$, et al. Health promotion in schools: polices and practices in Stockholm County, 1990. Scand J Soc Med. 1995; 23(1):39-46. doi: 10.1177 140349489502300108.

10. Kafatos I, Peponaras A, Linardakis M, Kafatos A. Nutrition education and Mediterranean diet: exploring the teaching process of a school-based nutrition and media education Project in Cretan primary schools. Public Health Nutr. 2004; 7(7): 969-75. doi: 10.1079/PHN2004565.

11. Schmitz BAS, Recine E, Cardoso GT, Silva JRM, Amorim NFA, Bernardon R, et al. A escola promovendo hábitos alimentares saudáveis: uma proposta metodológica de capacitação para educadores e donos de cantina. Cad Saúde Pública. 2008; 24(Supl 2):S312-S22. doi: 10.1590/S0102$311 \times 2008001400016$.
12. Manios Y, Moschandreas J, Hatzis C, Kafatos A. Health and nutrition education in primary schools of Crete: changes in chronic disease risk factors following a 6-year intervention programme. $\mathrm{Br} \mathrm{J}$ Nutr. 2002; 88:315-24. doi: 10.1079/BJN2002 672.

13. Focesi E. Educação em saúde na escola: o papel do professor. Rev Bras Saúde Esc. 1990; 1(2):4-8.

14. Brasil. Ministério da Saúde. Política nacional de alimentação e nutrição. $2^{a}$ ed. Brasília: Ministério da Saúde; 2003.

15. Componentes educativos de los programas para la promoción de la salud escolar. Rev Panam Salud Publica. 1997; 2(3):209-14. doi: 10.1590/\$1020-49 891997000900014.

16. World Health Organization. Expert consultation on diet, nutrition and the prevention of chronic diseases. Geneva; 2002 [cited 2002 Jan 28]. Available from: < http://www.fao.org/docrep/005/ AC911E/AC911E00.HTM>.

17. Barreto SM, Pinheiro ARO, Sichieri R, Monteiro CA, Batista Filho M, Schimidt MI, et al. Análise da estratégia global para alimentação, atividade física e saúde, da Organização Mundial da Saúde. Epidemiol Serv Saúde. 2005; 14(1):41-68.

18. Instituto Brasileiro de Geografia e Estatística. Pesquisa de orçamentos familiares 2002/2003: análise da disponibilidade domiciliar de alimentos e do estado nutricional no Brasil. Rio de Janeiro; IBGE; 2004.

19. Auld GW, Romaniello C, Heimendinger J, Hambidge C, Hambidge M. Outcomes from a school-based nutrition education program alternating special resource teachers and classroom teachers. J Sci Health. 1999; 69(10):403-8. doi:10.1111/j.1746-1561.1999.tb06358.x.

20. Pelicioni MCF, Torres AL. A escola promotora de saúde. São Paulo: Eixo promoção da Saúde; 2005. Série monográfica 12.

21. Lytle L, Fulkerson JA. Assessing the dietary environment: examples from school-based nutrition interventions. Public Health Nutr. 2002; 5(6A):893-9. doi:10.1079/PHN2002384.

22. Temporini ER. Percepção de professores do sistema de ensino do Estado de São Paulo sobre seu preparo em saúde do escolar. Rev Saúde Pública. 1988; 22(5):411-21. doi:10.1590/S0034-891019 88000500006 .

23. Friel S. Kelleher C, Campbell P, Nolan G. Evaluation of the nutrition education at primary school (NEAPS) programme. Public Health Nutr. 1999; 2(4): 549-55. doi:10.1017/S1368980099000737. 
24. Franco AC, Boog MCF. Relação teoria-prática no ensino de educação nutricional. Rev Nutr. 2007; 20(6):643-55. doi: 10.1590/S1415-S273200700 0600007.

25. Conteto IR, Randell JS, Basch CE. Review and analysis os evaluation measures used in nutrition education intervention research. J Nutr Educ Behav. 2002; 34(1):2-25. doi:10.1016/S1499-4046(06) 60220-0.

26. Oshaug A. Evaluation of nutrition education programmes: implications for programme planners and evaluators. In: Discussion papers of the FAO expert consultation on nutrition education for the puplie. Rome: FAO; 1987. p.151-78.

27. Costa EQ, Ribeiro VMB, Ribeiro ECO. Programa de alimentação escolar: espaço de aprendizagem e produção de conhecimento. Rev Nutr. 2001; 14(3): 225-9. doi:10.1590/S1415-52732001000300 009.

28. Poulain JP, Proença RPC. Reflexões metodológicas para o estudo das práticas alimentares Rev Nutr.
2003; 16(4):365-86. doi: 10.1590/S1415-52732 003000400001.

29. Davanço GM, Taddei JAAC, Gaglionone CP. Conhecimentos, atitudes e práticas de professores de ciclo básico, expostos e não exposto a curso de educação nutricional. Rev Nutr. 2004; 17(2): 177-84. doi: 10.1590/S1415-52732004000200 004.

30. Triches RM, Giugliani ERJ. Obesidade, práticas alimentares e conhecimentos de nutrição em escolares. Rev Saúde Pública. 2005; 39(4):541-7. doi: 10.1590/S0034-89102005000400004.

31. Rocha HHP. Educação escola e higienização da infância. Cad Cedes. 2003; 59 (23):39-56. doi: $10.1590 / \$ 1415-52732004000200004$.

Recebido em: 12/3/2008

Versão final reapresentada em: 2/3/2009 Aprovado em: 6/10/2009 


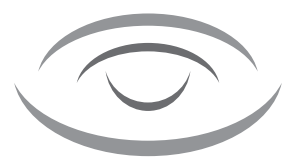

\section{ANEXOS}

Observatório de Políticas de Segurança Alimentar e Nutrição

Universidade de Brasília/Núcleo de Pesquisas em Políticas Públicas

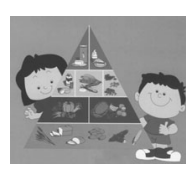

\section{ANEXO 1}

Instrumentos de avaliação dos alunos - pirâmide dos alimentos

Vamos encontrar os alimentos parecidos! Ligue os alimentos que são do mesmo grupo!

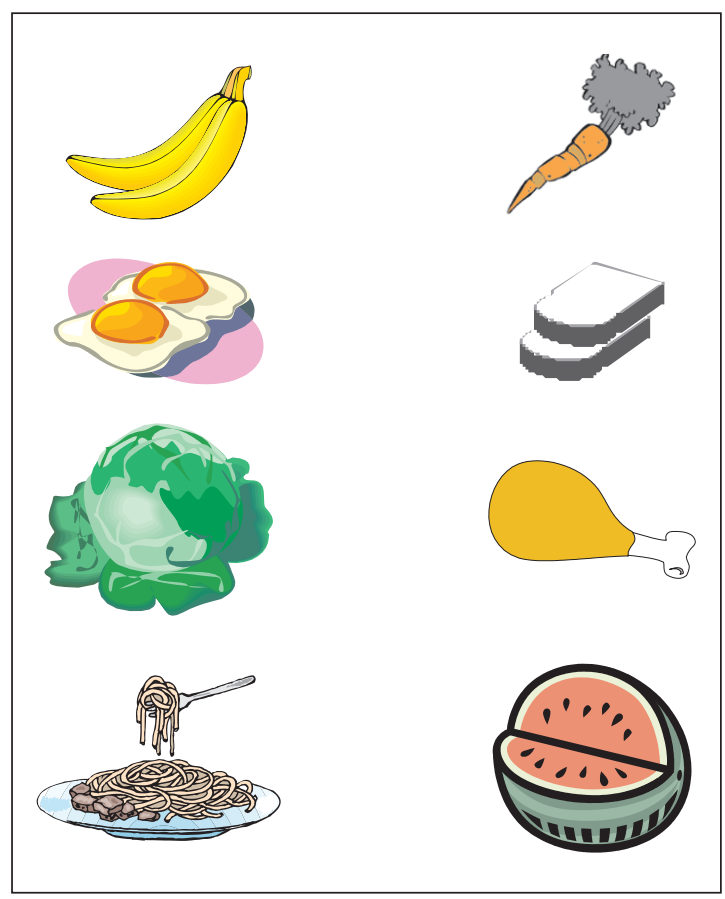

\section{ANEXO 2}

Vamos ordenar as figuras? Enumere as figuras de acordo com a sequência correta de eventos.

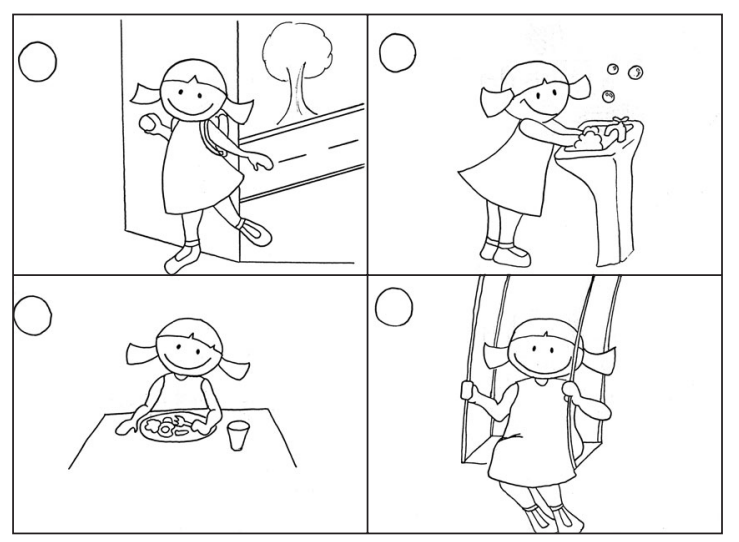

ANEXO 3

Questionário de avaliação com professores

De acordo com o seu conhecimento sobre alimentação saudável até o momento, marque nos espaços abaixo a letra $V$ para as frases que considerar como VERDADEIRAS e $F$ para as que considerar FALSAS.

1. ( ) É correto uma pessoa comer muito em uma refeição e diminuir bastante a quantidade das outras refeições do dia para compensar.

2. ( ) A alimentação saudável inclui a pessoa ter prazer e estar feliz ao comer.

3. ( ) Existe apenas um tipo de alimentação correta para todas as pessoas.

4. ( ) O milho faz parte do grupo das hortaliças.

5. ( ) De acordo com os grupos de alimentos, as frutas e as hortaliças são a base da alimentação.

6. ( ) As crianças nunca podem comer doces e biscoitos.

7. ( ) A higiene com os alimentos e na hora de comer faz parte da alimentação saudável.

8. ( ) Para uma pessoa ser saudável ela deve ser bem magrinha.

9. ( ) Fazer atividade física em exagero faz mal à saúde. 
